\title{
PERFIL LIPÍDICO EM CRIANÇAS E ADOLESCENTES COM EXCESSO DE PESO
}

\section{LIPID PROFILE IN OVERWEIGHT CHILDREN AND ADOLESCENTS}

\author{
Alessandra Teixeira Ramos ${ }^{1}$, Danielle Franklin de Carvalho ${ }^{1,2}$, \\ Nathalia Costa Gonzaga', Anajás da Silva Cardoso', \\ Juliana Andreia Fernandes Noronha', Maria Aparecida Alves Cardoso ${ }^{1}$
}

RESUMO

Objetivo: verificar alterações lipídicas e fatores associados em crianças e adolescentes obesos ou com sobrepeso. Método: estudo transversal realizado entre abril e novembro de 2009, incluindo 217 crianças e adolescentes, com excesso de peso, entre dois e 18 anos, usuários do Sistema Unico de Saúde, Campina Grande-PB. A classificação do estado nutricional, segundo o percentil, considerou: sobrepeso (85e"IMC <95), obesidade (95e"IMC<97) e obesidade grave (IMCe"97). Foram considerados alterados os valores de LDL-c e" $130 \mathrm{mg} / \mathrm{dL}$, colesterol HDL-c < $45 \mathrm{mg} / \mathrm{dL}$ e triglicerídeos e" 130mg/ dL. A análise estatística, realizada no SPSS 17.0, utilizou análise de variância e testes de qui-quadrado, adotando-se um intervalo de confiança de 95\%. Resultados: a maioria das crianças e adolescentes apresentava obesidade grave $(68,2 \%)$, que se mostrou associada ao sexo masculino $(R P=3,7)$ e à faixa etária entre dois e nove anos $(R P=3,2)$. A dislipidemia foi observada em $85,3 \%$ da população estudada, sendo mais prevalente $(88,9 \%)$ entre as crianças de dois a cinco anos. O HDL-c baixo foi a alteração mais frequente $(80,6 \%)$, e seu valor foi significativamente mais baixo entre os obesos graves $(p<0,005)$. Conclusões: a elevada prevalência de dislipidemia, observada desde a faixa pré-escolar, reforça a necessidade de se monitorar o perfil lipídico, na presença de obesidade ou sobrepeso, independentemente da idade.

Palavras-chave: obesidade; sobrepeso; dislipidemias; doença cardiovascular

\begin{abstract}
Background: to assess lipid profile alterations and associated factors in obese or overweight children and adolescent. Methods: cross-sectional study developed from April to November 2009, including 217 overweight children and adolescents, from 2 to 18 years old, users of the public health system, Campina Grande-PB. nutritional status was classified, according to percentile, as: overweight $\left(85 \mathrm{e}^{\prime \prime} \mathrm{bmi}<95\right)$, obese $\left(95 \mathrm{e}^{\prime \prime} \mathrm{bmi}<97\right)$ and severe obesity (bmie"97). it was considered abnormal the following values: Idl-c $e^{\prime \prime}$ $130 \mathrm{mg} / \mathrm{dl}$, hdl-c $<45 \mathrm{mg} / \mathrm{dl}$ and $\mathrm{tg} \mathrm{e}^{\prime \prime} 130 \mathrm{mg} / \mathrm{dl}$. statistical analysis included variance analysis and chi-square tests adopting a confidence interval of 95\%, using spss 17.0 . Results: the majority of the children and adolescents presented severe obesity $(68,2 \%)$ which was higher in boys ( $\mathrm{pr}=3,7)$ and in children from two to nine years old $(\mathrm{pr}=3,2)$. dyslipidemias were observed in $85,3 \%$ of the studied population, being more prevalent $(88,9 \%)$ in children from two to five years old. the low hdl-c was the most frequent alteration $(80,6 \%)$, and its value was significantly lower among those with severe obesity $(p<0,005)$. Conclusions: the high prevalence of dyslipidemia, observed since the pre-school age, reinforces the need to monitor the lipid profile, in the presence of obesity or overweight condition, independently of age.
\end{abstract}

Key words: obesity; overweight; dyslipidemias; cardiovascular disease.

1 Núcleo de Estudos e Pesquisas Epidemiológicas (NEPE) - Universidade Estadual da Paraíba (UEPB) - Endereço: Avenida das Baraúnas, 351 - Cidade Universitária - Prédio dos Mestrados - $2^{\circ}$ andar - Campina Grande-PB, CEP: 58429-500, Fone/Fax: (83) 3315-3300

2 Universidade Federal de Pernambuco - Endereço: Av. Prof. Moraes Rego, 1235 - Cidade Universitária, Recife - PE CEP: 50670-901 | Fone PABX: (81) 2126.8000

REALIZAÇÃO DO TRABALHO: Núcleo de Estudos e Pesquisas Epidemiológicas (NEPE) - Universidade Estadual da Paraíba (UEPB) - Endereço: Avenida das Baraúnas, 351 - Cidade Universitária - Prédio dos Mestrados - $2^{\circ}$ andar Campina Grande-PB, CEP: 58429-500, Fone/Fax: (83) 3315-3300

Pesquisa financiada pela Fundação de Apoio à Pesquisa do Estado da Paraíba-FAPESQ, através do edital 01/2008FAPESQ/PB-MCT/CNPq (Termo de Concessão nº 198/08)

Corepondência para: carlacamposmunizmedeiros@gmail.com

Como citar este artigo: Ramos AL et al. Lipid profile in overweight children and adolescents. Journal of Human Growth and Development 2011; 21(3): 780-788.

Artigo submetido em 06.12.10, aceito em 10.07.11. 


\section{INTRODUÇÃO}

A doença aterosclerótica é atualmente uma das principais causas de morbimortalidade no Brasil e no mundo. É multifatorial, podendo ter início silencioso na infância, com progressão durante a adolescência e a idade adulta ${ }^{1}$.

Recentemente, tem sido reconhecido que alterações dos níveis séricos de lipídeos na infância podem ser preditivas da ocorrência de doença cardiovascular em adultos. Isso se baseia no fenômeno de trilha (tracking), em que ocorre uma forte tendência das crianças manterem os mesmos percentis de colesterol até a vida adulta ${ }^{4}$. Além disso, a persistência de um perfil lipídico desfavorável aumenta o risco de eventos coronarianos, o que justifica a importância de adotar medidas de prevenção já na infância ${ }^{4,5}$.

O aumento, em níveis epidêmicos, do excesso de peso infantil, parece ser o responsável pelas mudanças negativas no perfil lipídico, precocemente representadas pelos níveis elevados de colesterol total (CT), triglicerídeos (TG), low density cholesterol (LDL-c) e níveis baixos de high density cholesterol (HDL-c) ${ }^{6}$.

Há evidências de que o processo de ocidentalização do estilo de vida está associado a uma tendência crescente da prevalência da dislipidemia. Por outro lado, observa-se um decréscimo naqueles países onde se instituiu programas de prevenção $0^{4}$.

Existem vários estudos sobre a prevalência de dislipidemia na infância, porém, no Brasil, poucos enfocaram a população de crianças e adolescentes com excesso de peso. Nestes, observam-se prevalências importantes de dislipidemia, que variam de $57,1 \%$ a $68,5 \%{ }^{9-12}$.

A identificação de crianças com fatores de risco para aterosclerose pode permitir intervenções precoces, reduzindo o processo aterosclerótico e, consequentemente, prevenindo ou retardando a ocorrência de doenças cardiovasculares.

Em 2005, a Sociedade Brasileira de Cardiologia publicou a I Diretriz de Prevenção da Aterosclerose na Infância e Adolescência ${ }^{13}$, recomendando mudança nos valores desejáveis dos lipídeos, com redução do valor do colesterol total e LDLc e aumento do HDL-c. Até o momento, nenhuma pesquisa foi realizada nesta população fazendo uso deste novo critério.

Portanto, o objetivo é verificar a ocorrência de alterações no perfil lipídico, bem como estudar os fatores associados, em crianças e adolescentes obesos ou com sobrepeso, usuários do Sistema Único de Saúde do município de Campina Grande-PB.

\section{MÉTODO}

Estudo transversal realizado entre abril e novembro de 2009, como parte de um projeto maior intitulado: "Prevalência de fatores de risco cardiometabólico entre crianças e adolescentes obesos ou com sobrepeso".

Foram incluídos crianças e adolescentes entre dois e 18 anos, com excesso de peso, usuários do Sistema Único de Saúde (SUS), do município de Campina Grande-PB. A captação foi realizada através da divulgação da pesquisa nas Unidades Básicas de Saúde, com apoio da secretaria de saúde do município. Os indivíduos eram encaminhados pelas equipes de saúde ao Centro de Obesidade Infantil (COI), implantado no Instituto de Saúde Elpídeo de Almeida, em Campina Grande-PB, especificamente para atender a demanda deste estudo. O COI é formado por pesquisadores e equipe multidisciplinar, composta por endocrinologista, nutricionista, psicóloga, enfermeira, assistente social e preparador físico.

No primeiro encontro, foi realizada uma triagem para avaliar se os indivíduos encaminhados atendiam aos critérios de inclusão do estudo. Do total de 246 casos, foram excluídos aqueles que, no momento da coleta de dados, fossem portadores de alguma doença ou que estivessem em uso de medicação que interferisse no metabolismo glicídico ou lipídico. Foram registradas 27 perdas por não comparecimento à coleta sanguínea e duas exclusões por uso de corticóide, 
perfazendo, no final, um total de 217 indivíduos acompanhados.

Os pais e/ou responsáveis eram informados sobre os objetivos e procedimentos do estudo e, em havendo interesse de participar, firmavam o compromisso através da assinatura do Termo de Consentimento Livre e Esclarecido (TCLE). Nesta ocasião, era ainda aplicado o primeiro questionário, que abordava questões socioeconômicas, de história pessoal e familiar; aferidas as medidas antropométricas e agendados os exames laboratoriais para determinação do perfil lipídico, realizados em até 15 dias após esta entrevista.

Os dados antropométricos (peso e estatura) foram coletados em duplicata, sendo considerado o valor médio das duas aferições. Para obtenção do peso, utilizou-se balança digital tipo plataforma (Welmy $®$ ) com capacidade para $200 \mathrm{~kg}$ e precisão de $0,1 \mathrm{~kg}$. A estatura foi aferida através de estadiômetro (Tonelli $\Re$ ), com precisão de $0,1 \mathrm{~cm}$. Durante a aferição, o indivíduo encontrava-se com roupas leves, sendo seguidos os procedimentos recomendados pela $\mathrm{OMS}^{14}$.

Para a classificação do estado nutricional, calculou-se o IMC, e conforme recomendações do Centers of Disease Control and Prevention (CDC) (2002) $)^{15}$ trabalhou-se, de acordo com os percentis, com as seguintes categorias: sobrepeso $\left(85 e^{\prime \prime}\right.$ IMC $\left.<95\right)$, obesidade $\left(95 e^{\prime \prime} I M C<97\right)$ e obesidade grave (IMCe" 97$)^{14}$.

Colesterol total, HDL-c e triglicerídeos foram avaliados através do método colorimétrico enzimático, em equipamento automático (Modelo BioSystems 310 ), de acordo com as recomendações do fabricante do kit Labtest $\AA$. A coleta sanguínea foi realizada após jejum de 10 a 12 horas, no Laboratório de Análises Clínicas da Universidade Estadual da Paraíba (LAC/ UEPB). Para o cálculo do LDL-C, foi utilizada a fórmula de Friedewald: LDL-C $=$ CT - HDL-C - TG/5, válida para valores de TG menores que $400 \mathrm{mg} / \mathrm{dL}$.

A prevalência de dislipidemia foi apresentada de acordo com a I Diretriz de Prevenção da Aterosclerose na Infância e Adolescência ${ }^{13}$, que estabelece os seguintes cortes para valores desejáveis: $\mathrm{CT}<150 \mathrm{mg} / \mathrm{dL}, \mathrm{LDL}-\mathrm{C}<100 \mathrm{mg} / \mathrm{dL}$, HDL-c e" 45mg/dL e TG < $100 \mathrm{mg} / \mathrm{dL}$; para valores limítrofes: CT $150-169 \mathrm{mg} /$ dL, LDL-c $100-129 \mathrm{mg} / \mathrm{dL}$ e TG $100-$ $129 \mathrm{mg} / \mathrm{dL}$; e valores alterados: CT $\mathrm{e}^{\prime \prime}$ $170 \mathrm{mg} / \mathrm{dL}$, LDL-c e" $130 \mathrm{mg} / \mathrm{dL}$, TG e" $130 \mathrm{mg} / \mathrm{dL}$ e HDL-c $<45 \mathrm{mg} / \mathrm{dL}$. Foram considerados casos de dislipidemia aqueles que apresentaram valores alterados de pelo menos um dos seguintes componentes: HDL-C, LDL-c e triglicerídeos, conforme a IV Diretriz Brasileira sobre Dislipidemias e Prevenção da Arterosclerose $^{16}$.

A análise estatística foi realizada através do SPSS, versão 17.0, utilizandose um intervalo de confiança de $95 \%$. Na análise descritiva, trabalhou-se com as categorias de estado nutricional e faixas etárias acima descritas. Para a realização dos testes de hipóteses, estas foram reagrupadas para a aplicação das análises de variância e testes do qui-quadrado.

A pesquisa foi aprovada pelo Comitê de Ética em Pesquisa da Universidade Estadual da Paraíba, sob o processo $\mathrm{n}^{\circ}$ 0040.0.133.000-08.

\section{RESULTADOS}

Das 217 crianças e adolescentes acompanhados, $64,1 \%$ eram do sexo feminino. A distribuição etária indicou que $12,4 \%$ tinham entre dois e cinco anos; $26,3 \%$ entre seis e nove e $61,3 \%$ entre 10 e 18 anos. Na caracterização socioeconômica, $19,9 \%$ tinham renda familiar correspondente a, no máximo, um salário mínimo; 38,9\% apresentavam entre um e dois; e $41,2 \%$, mais de dois. No que se refere à escolaridade materna, 37,3\% tinham nível médio e 14,3\%, superior.

A maioria dos indivíduos $(68,2 \%)$ encontrava-se com o IMC e" percentil 97, caracterizando obesidade grave; $18,9 \%$ estavam entre os percentis 95 e 97; e $12,9 \%$ na faixa de 85 e" IMC < 95. A obesidade grave mostrou-se associada ao sexo masculino (RP $=3,7-\mathrm{IC}_{95 \%}[1,7-$ $6,7]$ ) e à faixa etária entre dois e nove anos (RP $=3,2-$ IC $\left._{95 \%}[1,7-6,1]\right)$. 
A dislipidemia foi observada em $85,3 \%$ da população estudada: isoladamente, $43,8 \%$ tinham apenas HDL-c baixo; $1,8 \%$, LDL-c alto e 0,5\%, TG alterado. Quando avaliadas as interações entre os diferentes lipídeos, verificou-se $24,9 \%$ de HDL-c baixo em associação com o aumento dos triglicerídeos; $9,2 \%$ de alterações associadas entre HDL-c, TG e LDL-C; 4,6\% com as duas frações de colesterol simultaneamente alteradas e, por fim, $0,5 \%$ de LDL-c e TG aumentados.
A distribuição do perfil lipídico encontra-se na figura 1 . O HDL-c baixo foi a alteração mais frequente $(80,6 \%)$, enquanto que a prevalência do LDL-c alterado foi a menos $(14,7 \%)$. Os valores médios dos lipídeos, com exceção do LDL$c$, encontram-se fora dos valores recomendados na I Diretriz de Prevenção de Aterosclerose na Infância ${ }^{13}$. Observou-se, ainda, que o valor do HDL-c foi significativamente mais baixo entre os pacientes obesos graves (Tabela 1).

Tabela 1: Média e desvio padrão do colesterol total, HDL-C, LDL-c e TG de acordo com o sexo, estado nutricional e faixa etária de 217 crianças e adolescentes com sobrepeso ou obesidade, Campina GrandePB, 2009

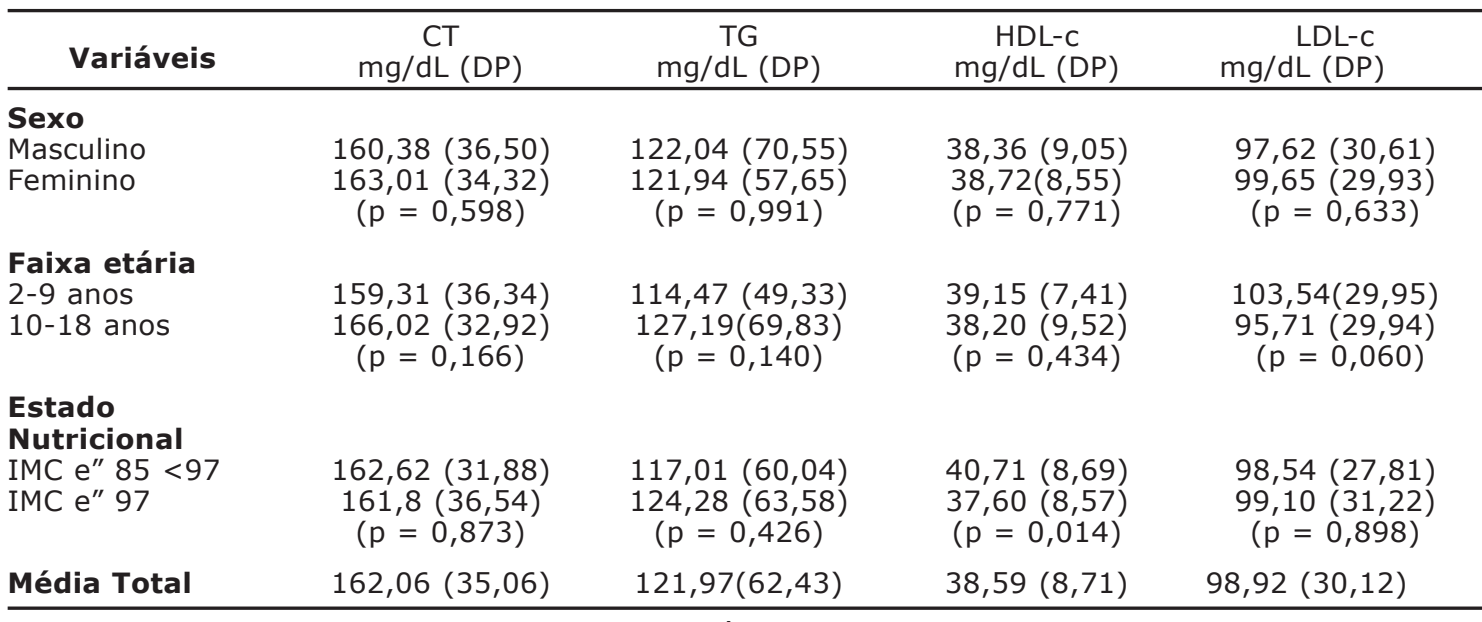

$\mathrm{CT}=$ Colesterol Total; $\mathrm{TG}=$ Triglicerídeos; HDL-C $=$ high density cholesterol; LDL-C = Iow density cholesterol; DP = Desvio-padrão; IMC = Índice de Massa Corporal; $\mathrm{p}=$ nível de significância de $5 \%$ (análise de variância).

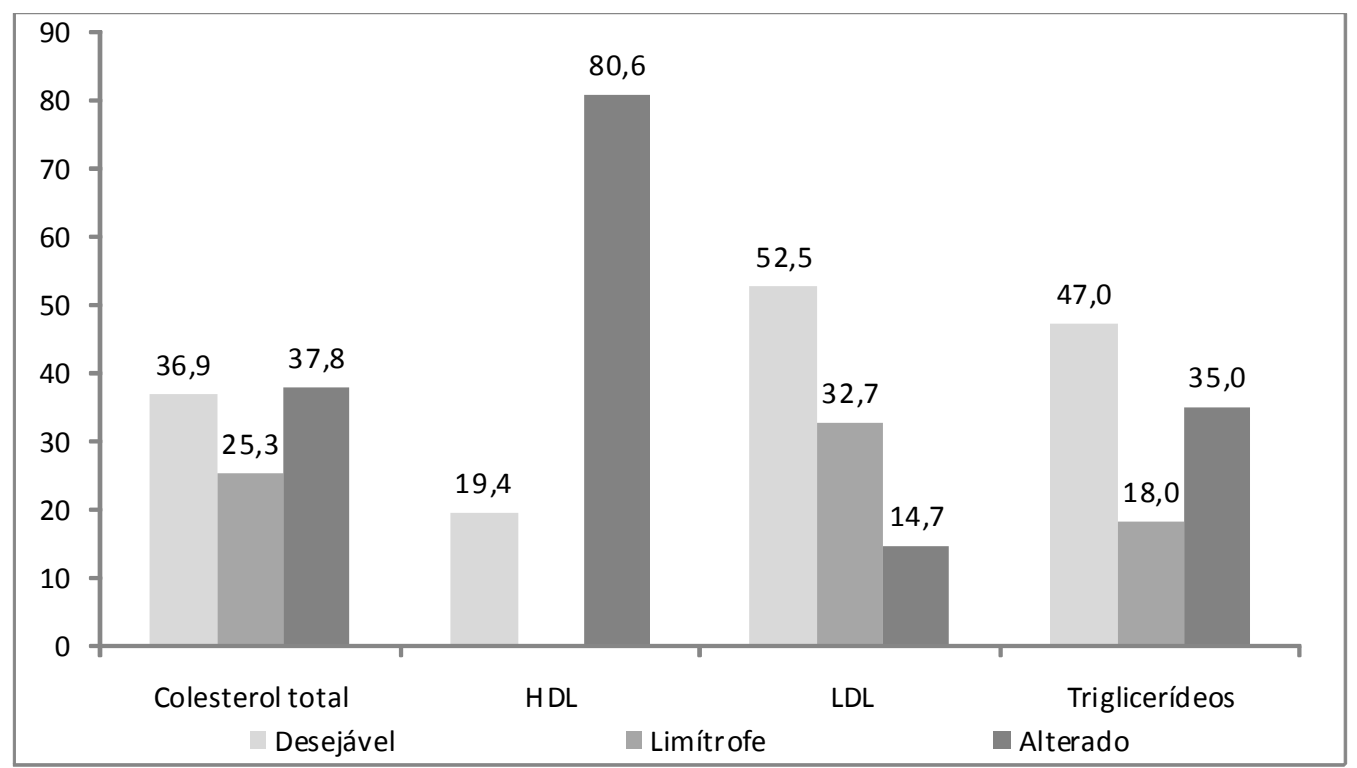

Figura 1: Valores desejáveis, limítrofes e alterados de colesterol total, HDL, LDL e triglicerídeos, de 217 crianças e adolescentes obesos ou com sobrepeso. Campina Grande-PB, 2009. HDL = high density cholesterol; $\mathrm{LDL}=$ Iow density cholesterol. 
A distribuição por faixa etária mostra que a alteração do CT foi mais frequente na faixa entre seis e nove anos, 0 LDL-c e o TG entre 10-18 anos e o HDL-C entre dois e cinco anos (Figura 2). Não foram observadas diferenças estatistica- mente significantes entre os valores alterados destas taxas (colesterol total, HDL-c, LDL-c e TG) para as diferentes faixas etárias, assim como da associação de dislipidemia com o estado nutricional, sexo ou faixa etária (Tabela 2 ).

Tabela 2: Distribuição da ocorrência e associação de dislipidemia nas 217 crianças e adolescentes estudados segundo a faixa etário, sexo e estado nutricional, Campina Grande-PB,2009

\begin{tabular}{|c|c|c|c|c|c|}
\hline \multirow[b]{2}{*}{ Variáveis } & \multicolumn{4}{|c|}{ Dislipidemia } & \multirow[b]{2}{*}{$\mathbf{p}$} \\
\hline & $\mathbf{n}$ & $\%$ & $\mathbf{n}$ & $\%$ & \\
\hline $\begin{array}{l}\text { Sexo } \\
\text { Feminino } \\
\text { Masculino }\end{array}$ & $\begin{array}{c}118 \\
67\end{array}$ & $\begin{array}{l}84,9 \\
85,9\end{array}$ & $\begin{array}{l}21 \\
11\end{array}$ & $\begin{array}{l}15,1 \\
14,1\end{array}$ & 0,841 \\
\hline $\begin{array}{l}\text { Faixa etária (em anc } \\
2-9 \\
10-18\end{array}$ & $\begin{array}{c}\text { 10s) } \\
70 \\
115\end{array}$ & $\begin{array}{l}83,3 \\
86,5\end{array}$ & $\begin{array}{l}14 \\
18\end{array}$ & $\begin{array}{l}16,7 \\
13,5\end{array}$ & 0,526 \\
\hline $\begin{array}{l}\text { Estado Nutricional ( } \\
85 \mathrm{e}^{\prime \prime} \text { IMC }<97 \\
\text { IMC e"97 }\end{array}$ & $\begin{array}{l}\text { (perc } \\
55 \\
130\end{array}$ & $\begin{array}{l}79,7 \\
87,8\end{array}$ & $\begin{array}{l}14 \\
18\end{array}$ & $\begin{array}{l}20,3 \\
12,2\end{array}$ & 0,116 \\
\hline
\end{tabular}

IMC = Índice de Massa Corporal; $p$ = nível de significância de 5\% (teste do qui-quadrado).

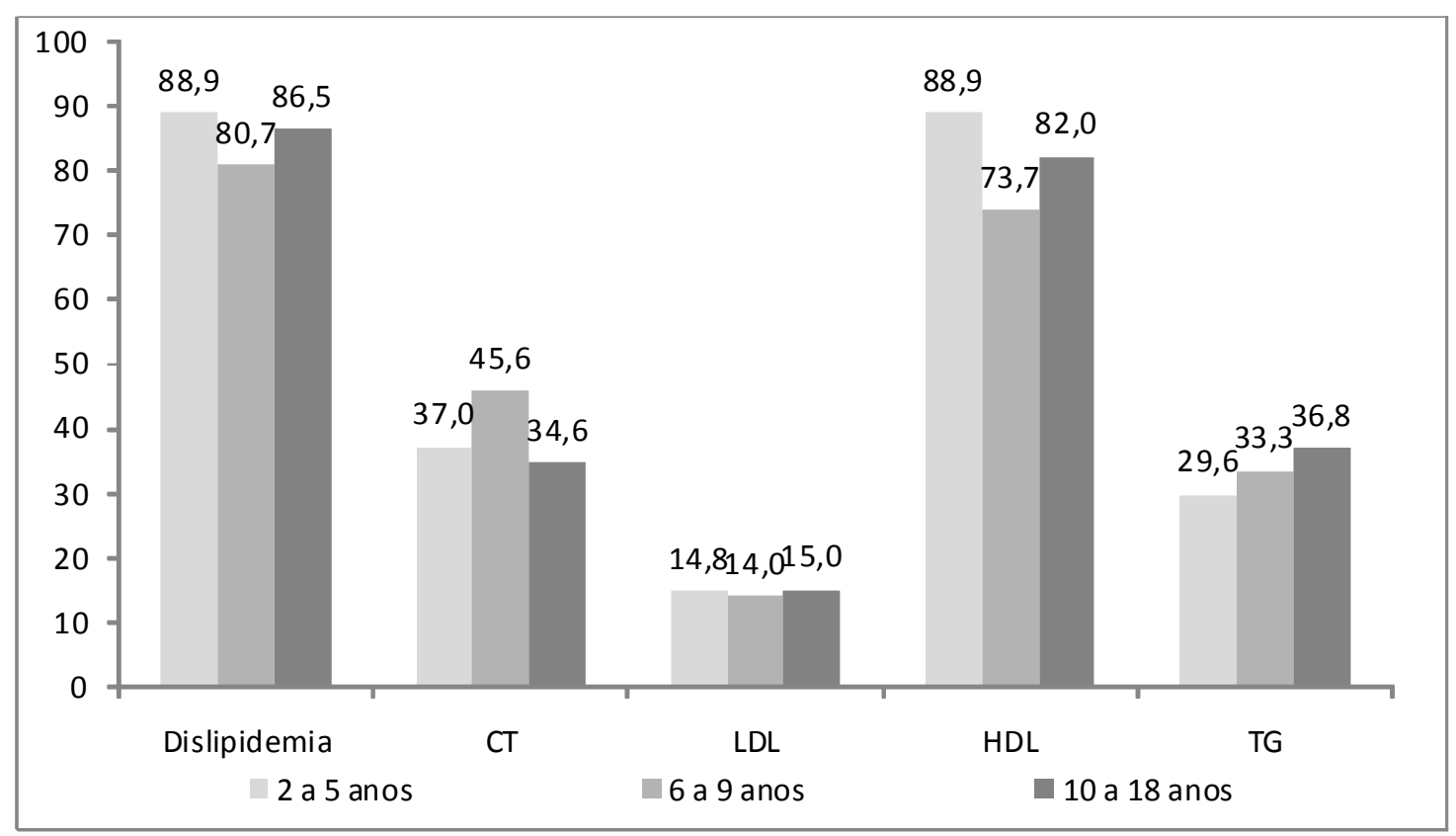

Figura 2: Distribuição da prevalência de dislipidemia, CT, LDL, HDL e TG, alterados, nas 217 crianças e adolescentes estudadas, segundo a faixa etária, Campina Grande-PB, 2009. CT = Colesterol Total; LDL-c = low density cholesterol; HDL-c = high density cholesterol; TG = Triglicerídeos.

\section{DISCUSSÃO}

Pesquisas científicas, em todo o mundo, mostram que os níveis de colesterol em crianças se associam diretamente à prevalência de doença coronariana nos adultos ${ }^{17-20}$. Esse fato reforça a preocupação em se reduzir os níveis médios de colesterol da população desde a infância, com o intuito de diminuir a frequência das complicações da aterosclerose.

O crescente aumento da prevalência de obesidade infantil e sua importân- 
cia enquanto fator de risco para dislipidemias consiste em um sério agravante para as doenças cardiovasculares. Estudos envolvendo adolescentes mostram que a obesidade relaciona-se com níveis adversos de lipídios e com lesões ateroscleróticas nas artérias coronarianas e aorta, em diferentes graus ${ }^{18,1}$.

No presente estudo, verificou-se uma prevalência de dislipidemia $(85,3 \%)$ superior àquela descrita na literatura para crianças e adolescentes brasileiros, que varia de $30,0 \%$ na população geral a $68,7 \%$ em crianças obesas ${ }^{19,12}$. Estudo anterior, desenvolvido no mesmo município, com adolescentes de 14 a 18 anos, não registrou casos de obesidade e a prevalência de sobrepeso foi de $14,4 \%$. Com relação à dislipidemia, verificou-se uma prevalência elevada $(66,7 \%)$, sendo o HDL-c baixo a alteração mais encontrada $(56,7 \%)^{21}$. É importante destacar que no estudo referido foi utilizado um ponto de corte, para o valor desejável, inferior $\left(e^{\prime \prime} 35 \mathrm{mg} / \mathrm{dL}\right.$ ) àquele adotado neste.

Ressaltam-se, nos resultados da presente pesquisa, a alta prevalência de obesidade grave $(68,2 \%)$ e o fato de que a dislipidemia foi encontrada em todas as faixas etárias, sendo mais frequente entre dois e cinco anos (88,9\%), seguida pela faixa de 10 a 18 anos, com $86,5 \%$. Ribas et al. ${ }^{20}$, estudando uma população entre seis e 19 anos, encontraram resultados semelhantes, sendo registrada a maior prevalência de dislipidemia na faixa entre 10 e 15 anos.

Freedman et al. ${ }^{17}$ constataram que o risco de hipertrigliceridemia parece ser maior nas crianças mais jovens, enquanto que o risco de hipercolesterolemia é maior nos adolescentes. Esses achados podem ser resultantes das variações dos níveis de lipídios e lipoproteínas séricas durante o crescimento e desenvolvimento, quando se observam duas fases de aumento expressivo: até o $2^{\circ}$ ano de vida e durante a maturação sexual ${ }^{5}$.

Estudo realizado em São Paulo, envolvendo crianças com história familiar de doença coronariana precoce, apontou que $38,5 \%$ apresentavam dislipidemia, valor que se elevou para $57,1 \%$ naqueles que tinham obesidade ou sobrepeso. É possível que essa menor prevalência, em relação ao nosso estudo, possa ser atribuída aos menores valores de referência adotados para o corte de HDL-c alterado utilizado pelos autores $^{11}$.

A associação entre massa corporal e perfil lipídico pode ser explicada pela ativação da via da cinase AMP-dependente, induzida pelo aumento da insulina e da leptina, bem como pela redução da ativação da adiponectina, que leva ao aumento da oxidação dos ácidos graxos. A adiponectina tem uma associação positiva com o aumento da sensibilidade à insulina e com os níveis de HDL-c e negativa com os níveis de triglicerídeos ${ }^{6}$. Esse fato foi constatado por estudos que mostraram, como principal causa de dislipidemia associada à obesidade, as elevações leves a moderadas do TG e diminuição do HDL-c ${ }^{9,12}$.

Prevalências de cerca de $50 \%$ de dislipidemia foram descritas em crianças com índice de massa corporal acima do percentil 99 para a idade ${ }^{6}$ e, em adolescentes, se observou uma associação linear positiva entre valores de IMC e os de colesterol total e LDL-C ${ }^{21}$. Na presente pesquisa, o IMC igual ou acima do percentil 97 mostrou-se predominantemente associado aos valores alterados de HDL-C, ao invés do LDL-c.

O HDL-c, lipoproteína que atua como fator protetor contra as doenças cardiovasculares, foi a alteração lipídica mais prevalente neste estudo $(80,6 \%)$. Este achado foi observado, embora em proporções menores, por outros autores, variando entre $29,5 \%$ e $68,7 \%{ }^{10,5}$. É importante ressaltar que os autores estudaram populações gerais de crianças e adolescentes, independentemente do estado nutricional, e com diferentes pontos de corte.

Níveis aumentados de HDL-c diminuem o risco relativo para a DCV, pela habilidade deste realizar o transporte reverso do colesterol e prevenir a oxidação e agregação das partículas de LDL-c na parede arterial, diminuindo o potencial aterogênico desta lipoproteína. Apesar de algumas pesquisas considerarem o aumento do colesterol total como fa- 
tor de risco cardiovascular, o LDL-c é considerado o principal preditor das DCV, sendo o maior alvo da intervenção médica $^{5}$. Nesse sentido, vale ressaltar o resultado de um estudo recentemente publicado, onde o colesterol total aumentado na infância não estava associado ao aumento do risco por morte endógena prematura 22,23 .

A prevalência de CT elevado foi encontrada em $37,8 \%$ da população estudada, situando-se dentro da faixa observada na literatura. No Brasil, a prevalência de hipercolesterolemia, em crianças e adolescentes da rede escolar, situa-se em torno de $35,0 \%$, quando o critério adotado é o CT superior a 170 $\mathrm{mg} / \mathrm{dL}^{24}$, corte semelhante ao adotado neste estudo.

Pereira et al..22, avaliando crianças e adolescentes de Itapetininga-SP, classificadas por estado nutricional, concluíram que a obesidade infantil determinou uma maior chance de ocorrência de níveis elevados de colesterol total, LDL-c e triglicérides, quando comparado às crianças eutróficas, fato não observado com relação ao HDL-c.

A hipertrigliceridemia esteve presente em 35\% dos indivíduos acompanhados, prevalência idêntica à observada por Lima et al.. ${ }^{10}$, porém inferior àquela de Valverde et al..12 (67,6\%). Apesar do TG não ser considerado um fator de risco independente para doença coronariana, seu excesso pode acarretar a formação de partículas pequenas e densas de LDL-c e/ou a diminuição dos níveis de $\mathrm{HDL}-\mathrm{C}^{9}$, fato este observado no presente estudo, em que a alteração do TG associado ao HDL-c esteve presente em $24,9 \%$ dos casos. Já na forma isolada, só foi registrada alteração em $0,5 \%$ dos indivíduos, achados semelhante ao de Romaldini et al..11, que encontraram uma frequência de 0,9\%, em crianças e adolescentes com história familiar de doença coronariana precoce.

O LDL-c foi a alteração lipídica menos frequente $(14,7 \%)$, porém é importante ressaltar que as crianças obesas parecem ter um maior percentual de LDLC de padrão $B$, partículas menores e mais aterogênicas, do que as crianças com peso normal para a estatura, aspecto não avaliado neste estudo ${ }^{13}$.

A prevalência da alteração do LDLc foi maior do que aquelas encontradas em estudos realizados nas escolas do estado de Pernambuco ${ }^{9}(10,0 \%)$ e de Florianópolis ${ }^{24}(6,0 \%)$, mas menor do que a encontrada por Lima et al. ${ }^{10} \mathrm{em}$ meninos obesos (50\%).

É importante destacar que, neste estudo, os valores médios dos lipídeos, com exceção do LDL-c, não se encontram dentro dos valores recomendados na I Diretriz de Prevenção de Aterosclerose na Infância. As médias do colesterol total $(162 \mathrm{mg} / \mathrm{dL})$ e LDL-c $(99 \mathrm{mg} / \mathrm{dL})$ aproximaram-se dos achados em pesquisas envolvendo crianças e adolescentes escolares realizadas por Giuliano et al. ${ }^{24}$, cujos valores foram de $162 \mathrm{mg} / \mathrm{dL}$ e de $92 \mathrm{mg} / \mathrm{dL}$, respectivamente; e por Moura et al..25, com CT de $160 \mathrm{mg} / \mathrm{dL}$ e LDL-c de $96 \mathrm{mg} / \mathrm{dL}$. Já os valores médios do TG $(122 \mathrm{mg} / \mathrm{dL})$ e HDL-c $(39 \mathrm{mg} / \mathrm{dL})$ foram diferentes aos encontrados nessas duas pesquisas, sendo na primeira o valor médio de TG $93 \mathrm{mg} / \mathrm{dL}$ e HDL-c $53 \mathrm{mg} / \mathrm{dL}$ e na segunda de $79 \mathrm{mg} / \mathrm{dL}$ e de $49 \mathrm{mg} /$ $\mathrm{dL}$, respectivamente ${ }^{24,25}$. Esses achados fortalecem a constatação de que as principais alterações do perfil lipídico nas crianças obesas ou com sobrepeso envolvem o TG e o HDL-c.

Alguns estudos epidemiológicos mostram que os níveis de lipoproteínas e lipídeos são mais elevados no gênero feminino e independem da idade ${ }^{5,20}$. No nosso estudo, a maior frequência de dislipidemia, apesar de não ser estatisticamente significante, foi observada no sexo masculino. Isto pode ser explicado devido à associação, na população estudada, da obesidade grave com o sexo masculino ( $R P=3,7)$.

A literatura mostra, ainda, que a prevalência de dislipidemia pode variar de acordo com a população estudada, pelos valores dos cortes adotados para valores desejáveis ou normais, bem como por fatores educacionais, culturais e genéticos ${ }^{24}$. Essas constatações nos remetem a uma das limitações deste estudo, que reside no fato de que os diferentes critérios adotados, assim como as dife- 
rentes populações estudadas, dificultaram as comparações. No entanto, é importante ressaltar que não encontramos estudos anteriores envolvendo crianças e adolescentes com excesso de peso, que tenham incluído o número de casos e a ampla faixa de idade como esta aqui enfocada.

Finalizando, é importante considerar que, mesmo não se tratando de estudo de base populacional, estes resultados refletem, de certa forma, o perfil da população de crianças e adolescentes obesos atendidos pelo SUS no município.

Assim, a elevada prevalência de dislipidemia na população estudada, desde a faixa pré-escolar, contribui para levantar evidências sobre a necessidade de se utilizar o sobrepeso ou obesidade como critério para avaliação do perfil lipídico, independentemente da idade. Além disso, é importante ressaltar que a dislipidemia é apenas umas das compli-

\section{REFERÊNCIAS}

1. McMahan CA, Gidding SS, Malcon GT, Tracy RE, Strong JP, McGill HC Jr. Pathobiological determinants of atherosclerosis in youth risk scores are associated with early and advanced atherosclerosis. Pediatrics. 2006; 118: 1447-55.

2. Pereira JC, Barreto SM, Passos VMA. Perfil de risco cardiovascular e autoavaliação da saúde no Brasil: estudo de base populacional. Rev Panam Salud Publica. 2009; 25(6): 491-8.

3. World Health Organization (WHO). Preventing Chronic Diseases - A Vital Investments. Geneva, 2005. 182 p.

4. Bridger T. Childhood obesity and cardiovascular disease. Paediatr Child Health. 2009; 14(3): 177-182.

5. Rabelo LM. Fatores de risco para doença aterosclerótica na adolescência. J Pediatr (Rio J). 2001; 77(2): s153-s64.

6. Weiss R, Dziura J, Burgert TS, Tamborlane WV, Taksali SE, Yeckel cações associadas à obesidade que, na sua forma grave, mostrou-se associada à faixa etária entre dois e cinco anos.

A implementação de programas de prevenção bem como a detecção e intervenção precoce das complicações associadas à obesidade, em particular, as dislipidemias, permitirão a redução do risco cardiovascular nesta população, que compõe parte significante da população geral. Dessa forma, estará se contribuindo para a confirmação do novo modelo de saúde, que preconiza proteção e promoção da saúde e prevenção de doenças.

\section{AGRADECIMENTOS}

À Fundação de Apoio à Pesquisa do Estado da Paraíba - FAPESQ, pelo financiamento através do edital 01/2008FAPESQ/PB-MCT/CNPq (Termo de Concessão no 198/08).

CW et al. . Obesity and the metabolic syndrome in children and adolescents. N Engl J Med. 2004; 350(23): 2362-74.

7. Daniels SR, Arnett DK, Eckel RH, Gidding SS, Hayman LL, Kumanyika $S$ et al.. Overweight in Children and Adolescents: Pathophysiology, Consequences, Prevention, and Treatment. Circulation. 2005; 111: 1999-2012.

8 Centers of Disease Control and Prevention (CDC). Prevalence of abnormal lipid levels among youths - United States, 1999-2006. MMWR. 2010; 59(2): 29-33.

9. Carneiro JRI, Kushnir MC, Clemente ELS, Brandão MG, Gomes MB. Obesidade na adolescência: fator de risco para complicações clínico-metabólicas. Arq Bras Endocrinol Metab. 2000; 44(5): 390-6.

10. Lima SC, Arrais RF, Almeida MG, Souza ZM, Pedrosa LF. Perfil lipídico e peroxidação de lipídeos no plasma em crianças e adolescentes com 
sobrepeso e obesidade. J Pediatr (Rio J). $2004 ; 80(1): 23-8$.

11. Romaldini $\mathrm{CC}$, Issler $\mathrm{H}$, Cardoso $A L$, Diament J, Forti N. Fatores de risco para aterosclerose em crianças e adolescentes com história familiar de doença arterial coronariana prematura. J Pediatr (Rio J). 2004; 80(2): 135-40.

12. Valverde MA, Vitolo MR, Patin RV, Escrivão MAMS, Oliveira FLC, AnconaLopez F. Investigação de alterações do perfil lipídico de crianças e adolescentes obesos. Arch Latinoam Nutr. 1999; 49: 338-43.

13. Giuliano ICB, Caramelli B, Pellanda L, Duncan B, Mattos S, Fonseca FAH et al.. I Diretriz de Prevenção da Aterosclerose na Infância e na Adolescência. Arq. Bras. Cardiol. [online]. 2005, 85(6): 3-36.

14. World Health Organization - WHO. Physical Status: the study and interpretation of anthropometry. WHO Technical Report Series n. 854. Geneva: WHO, 1995.

15. Centers of Disease Control and Prevention (CDC) table for calculated body mass index values for selected highs and weights for ages 2 to 20 years. Developed by the National Center for Health Statistc in collaboration with the National Center for Chronic Disease Prevention and Health Promotion, 2000. Publicado em maio de 2002, modificado 20/04/ 2001. Disponível em: htpp:// www.cdc.gov/growthcharts.

16. Sposito AV, Caramelli $B$, Fonseca FAH, Bertolami MC, Afiune NA, Souza AD et al.. IV Diretriz Brasileira sobre Dislipidemias e Prevenção da Aterosclerose do Departamento de Aterosclerose da Sociedade Brasileira de Cardiologia. Arq Bras Cardiol. 2007; 88(1): 1-19.
17. Freedman DS, Khan LK, Dietz WH, Srinivasan SR, Berenson GS. Relationship of childhood obesity to coronary heart disease risk factors in adulthood: the Bogalusa Heart Study. Pediatrics. 2001; 108(3): 712-8.

18. Hong MY. Atherosclerotic Cardiovascular Disease Beginning in Childhood. Korean Circ J. 2010; 40: 1-9.

19. França E, Alves JGB. Dislipidemia entre crianças e adolescentes de Pernambuco. Arq Bras Cardiol. 2006; 87(6): 722-7.

20. Ribas AS, SILVA LCS. Dislipidemia em escolares na rede privada de Belém. Arq. Bras. Cardiol. 2009; 92(6): 44651.

21. Carvalho DF, Paiva AA, Melo ASO, Ramos AT, Medeiros JS, Medeiros CCM et al.. Perfil lipídico e estado nutricional de adolescentes. Rev. bras. Epidemiol. 2007; 10(4): 4918.

22. Pereira A, Guedes AD, Verreschi ITN, Santos RD, Martinez TLR. A Obesidade e sua Associação com os Demais Fatores de Risco Cardiovascular em Escolares de Itapetininga, Brasil. Arq Bras Cardiol. 2009; 93(3): 253-60.

23. Franks $\mathrm{PW}$, Hanson $\mathrm{R} L$, Knowler WC, Sievers, ML, Bennett PH, Looker HC, MB. Childhood Obesity, Other Cardiovascular Risk Factors, and Premature Death. N Engl J Med. 2010; 362: 485-93.

24. Giuliano IC, Coutinho MS, Freitas SF, Pires MM, Zunino JN, Ribeiro RQ. Lípides séricos em crianças e adolescentes da rede escolar de Florianópolis - Estudo Floripa Saudável 2040. Arq Bras Cardiol. 2005; 85(2): 85-91.

25. Moura EC, de Castro CM, Mellin AS, de Figueiredo DB. Perfil lipídico em escolares de Campinas, SP, Brasil. Rev Saúde Pública. 2000; 34(5): 499-505. 